\title{
Simulations of fine structures on the zero field steps of Josephson tunnel junctions
}

\author{
Scheuermann, M.; Chi, C. C.; Pedersen, Niels Falsig; Chang, Jhy-Jiun; Chen, J. T.
}

Published in:

Applied Physics Letters

Link to article, DOI:

$10.1063 / 1.96939$

Publication date:

1986

Document Version

Publisher's PDF, also known as Version of record

Link back to DTU Orbit

Citation (APA):

Scheuermann, M., Chi, C. C., Pedersen, N. F., Chang, J-J., \& Chen, J. T. (1986). Simulations of fine structures on the zero field steps of Josephson tunnel junctions. Applied Physics Letters, 48(2), 189-191.

https://doi.org/10.1063/1.96939

\section{General rights}

Copyright and moral rights for the publications made accessible in the public portal are retained by the authors and/or other copyright owners and it is a condition of accessing publications that users recognise and abide by the legal requirements associated with these rights.

- Users may download and print one copy of any publication from the public portal for the purpose of private study or research.

- You may not further distribute the material or use it for any profit-making activity or commercial gain

- You may freely distribute the URL identifying the publication in the public portal

If you believe that this document breaches copyright please contact us providing details, and we will remove access to the work immediately and investigate your claim. 


\title{
Simulations of fine structures on the zero field steps of Josephson tunnel junctions
}

\author{
M. Scheuermann and C. C. Chi \\ IBM Thomas J. Watson Research Center, P.O. Box 281, Yorktown Heights, New York 10598 \\ N. F. Pedersen \\ Physics Laboratory I, The Technical University of Denmark, DK-2800 Lyngby, Denmark \\ Jhy-Jiun Chang and J. T. Chen \\ Department of Physics and Astronomy, Wayne State University, Detroit, Michigan 48202
}

(Received 26 August 1985; accepted for publication 11 November 1985)

\begin{abstract}
Fine structures on the zero field steps of long Josephson tunnel junctions are simulated for junctions with the bias current injected into the junction at the edges. These structures are due to the coupling between self-generated plasma oscillations and the traveling fluxon. The plasma oscillations are generated by the interaction of the bias current with the fluxon at the junction edges. On the first zero field step, the voltages of successive fine structures are given by $V_{n}=\hbar / 2 e\left(2 \omega_{p} / n\right)$, where $n$ is an even integer.
\end{abstract}

It is well known that fluxon propagation is responsible for the zero field steps found on the current-voltage characteristics of long tunnel junctions. ${ }^{1-3}$ Recently, several careful experimental studies have revealed a set of fine structures (FS's) superimposed on the zero field steps. ${ }^{4,5}$ It has been suggested that the FS's are a manifestation of the interaction between the traveling fluxon and other modes which may be present in the junction.

An example of these fine structures is shown in Fig. 1(a). Initial speculation ${ }^{4}$ related these FS's to a coupling between the traveling fluxon and cavitylike oscillations. Although it is possible to assign most of the FS's to a specific harmonic or subharmonic of a cavity mode, there is no satisfactory answer to the question why some of the lower harmonics of the cavity modes are not present. Later, Pedersen and Welner ${ }^{5,6}$ suggested that these steps may be related to the interaction of the traveling fluxon with plasma oscillations generated by the reflection of the fluxon at the edges of the junction. They argue that strong coupling occurs when the fluxon makes one round trip across the junction during an integer $(n)$ number of plasma oscillations. This implies for the first zero field step FS's should occur at voltages given by

$$
V_{n}=\frac{\hbar}{2 e}\left(\frac{2 \omega_{p}}{n}\right)
$$

This equation seems to fit the experimental data better. However, up until this time, no theoretical calculations have been done to reproduce these fine structures. In this letter we show that the FS's on the zero field step can be reproduced very well using numerical simulation for proper conditions. The origin of the FS's and the conditions necessary to be observed by them are discussed.

Simulations are performed using the perturbed SineGordon equation for one-dimensional junctions with surface losses neglected. In normalized form, this equation can be written in the form

$$
\phi_{x x}-\phi_{t t}-\alpha \phi_{r}=\sin \phi-\eta,
$$

where $\phi$ is the phase difference between the two superconducting films. The damping parameter $\alpha$ is given by
$\alpha=G(\hbar / 2 e J C)$, where $G, J$, and $C$ are the quasiparticle conductance, critical current, and capacitance per unit length. In this equation, the spatial coordinate $x$ is measured in units of $\lambda_{J}=\left(\hbar / 2 \mu_{0} e d J\right)^{1 / 2}$ while the temporal coordinate is measured in units of the inverse plasma frequency $\omega_{0}^{-1}=(\hbar C / 2 e J)^{1 / 2}$. The parameter $\eta$ represents the bias current distribution normalized to the critical current per unit length of the junction. Two current distributions are used in the simulations discussed below. For uniform bias current distribution $\eta=I_{\mathrm{dc}} / I_{0}$, where $I_{\mathrm{dc}}$ is the bias current and $I_{0}$ is the maximum zero voltage current. The boundary conditions for this case are $\phi_{x}(0, t)=\phi_{x}(L, t)=0$, which correspond to zero external magnetic field. For edge current injection the bias current distribution $\eta$ is a sum of two delta functions at the edges of the junction, which is mathematically equivalent to imposing the boundary conditions $\phi_{x}(0, t)=-\phi_{x}(L, t)=I_{\mathrm{dc}} / 2 J \lambda_{J}$ on $\mathrm{Eq}$. (2) with $\eta=0$.
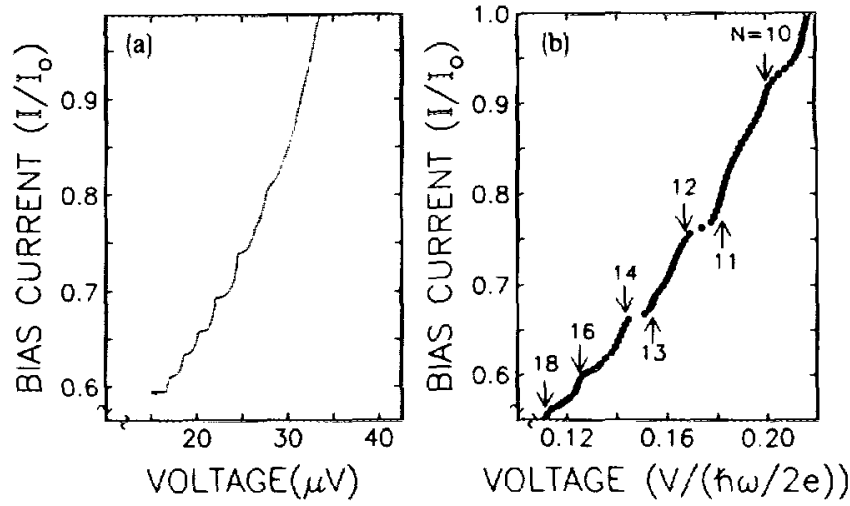

FIG. 1. (a) Current-voltage characteristic showing the lower portion of the first zero field step of $\mathrm{Sn}-\mathrm{SnO} \mathrm{x}-\mathrm{Sn}$ Josephson tunnel junction having overlap geometry with length $0.95 \mathrm{~mm}$, width $0.14 \mathrm{~mm}$, and with the parameters $j$, $\lambda_{J}$, and $\omega_{0}$ estimated from experimental results to be $1.3 \times 10^{2} \mathrm{~A} / \mathrm{cm}^{2}, 43$ $\mu \mathrm{m}$, and $90 \mathrm{GHz}$, respectively. This curve was taken at $T=1.65 \mathrm{~K}$ in zero magnetic field. The horizontal arrow indicates switching to the zero voltage state. (b) Dotted curve shows the current-voltage characteristics of the first zero field step as calculated using numerical simulations. Parameters used for this calculation are $L / \lambda_{J}=20, a=0.06$, and nonuniform (edge) current distribution. Vertical arrows and the associated $\boldsymbol{n}$ indicate the voltages $V_{n}$ of Eq. (1). 
These boundary conditions represent the self-induced magnetic fields due to the bias current at the edges of the junction.

Figure 1(b) shows a numerical simulation of the first zero field step for a junction with parameters $L / \lambda_{J}=20$, $\alpha=0.06$, and $\eta$ corresponding to edge injection. Each point on this curve represents the calculated average voltage for the given bias current. The structures on this curve are similar to the experimental FS's shown in Fig. 1 (a). These structures are absent in the analytic perturbative solutions ${ }^{7.8}$ of Eq. (2). Numerical simulations show large oscillations are generated at the edge of the junction after the fluxon is reflected from the edge as shown in Fig. 2. Although these oscillations are not completely uniform in space, the frequency is very close to the plasma frequency of the junction suggesting the plasma oscillations generated by the system may be responsible for the FS's. It is well known that plasma oscillations are generated when a fluxon is accelerated. For a junction with edge injection, the large current spikes near the junction edge provide an impulselike energy input to the fluxon causing a rapid increase in its velocity. To check that the plasma oscillations generated at the edges of the junction are indeed responsible for the FS's and the nonuniform current distribution plays a major role, we also performed numerical simulations for a junction with uniform bias current. In that case, the plasma oscillations are much smaller and virtually no FS's are found.

Experimentally, junctions showing well-defined FS's have been junctions of overlap geometry without superconducting ground planes. There are many reasons to believe that the overlap geometry without a ground plane does not provide uniform current distribution. One of the experimental evidences showing a nonuniform distribution is that the zero voltage current is much lower than one would expect for junctions with a uniform current distribution. Other evidence indicating that the bias current is confined toward the edges of the junction is provided by laser scanning experiments ${ }^{9}$ which can directly probe the supercurrent distribution. In most overlap junctions, the actual current distribu-

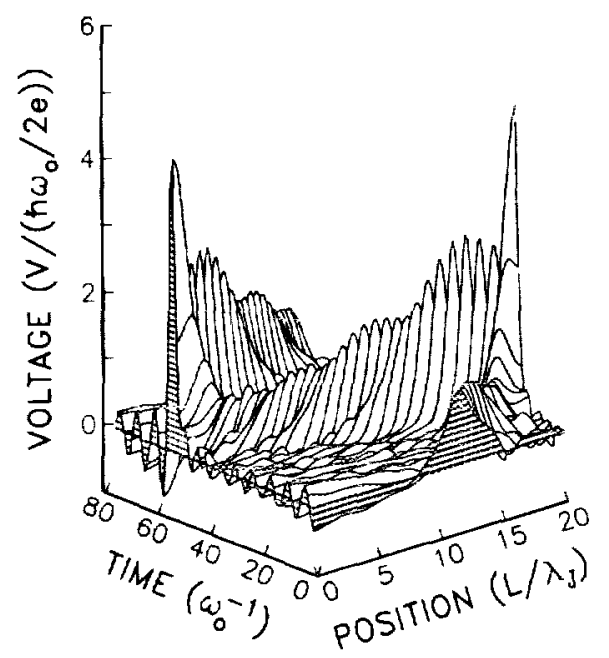

FIG. 2. (a) Simulation for $L / \lambda_{s}=20, \alpha=0.06, I / I_{0}=0.8$ for nonuniform (edge) current injection. tion is likely to be entirely edge injection. However, the nonuniform component of the distribution is primarily responsible for the FS's in these simulations. It should be pointed out that a nonuniform current distribution is not the only source of plasma oscillations. Numerous other inhomogeneities and effects can cause plasma oscillations by perturbing the fluxon velocity.

The voltage positions $V_{n}$ given by Eq. (1) are indicated by the arrows in Fig. 1(b). For the current range shown, there is a set of well defined structures at voltages labeled by even value of $n$. For odd values of $n$, there is some indication of very weak structures. The larger FS's labeled by even values of $n$ can be understood by the following. If the fluxon travels across the junction once during an integer number of plasma oscillations, it will arrive at the opposite edge in phase with the plasma oscillations generated during the previous reflection at that edge. This corresponds to a round trip time equal to an even number of plasma oscillations and strong coupling. On the other hand, if the vortex travels across the junction once during $n+1 / 2$ plasma oscillations, it will arrive at the opposite edge out of phase with the plasma oscillations generated by the previous reflection. This would correspond to a round trip time equal to an odd number of plasma oscillation and weak coupling. Using even $n$ to label successive FS's is important since the plasma frequency can be determined by using Eq. (1) to fit the experimental FS's. The resultant plasma frequency would be off by a factor of 2 if successive FS's are labeled using consecutive integers. Figure 3 demonstrates the graphical construction used to determine the plasma frequency. These data are taken from the experimental curve shown in Fig. 1(a). The plasma voltage ( $\hbar \omega_{p} / 2 e$ ) is found directly from the graph for $n=2$. To determine the accuracy of the technique, a similar construction was used to determine the plasma frequency of the simulated curve of Fig. 1(b). The value found was within $4 \%$ of the set value. Using this technique to measure the plasma frequency is particularly useful for long junctions with a nonuniform current distribution since other parameters $\left(\bar{c}\right.$ and $\left.\lambda_{J}\right)$ cannot be measured with a good accuracy.

In this letter we report on computer simulations which show that under the appropriate conditions a well defined set of FS's can be observed on the first zero field step. These structures are shown to be generated by the interaction

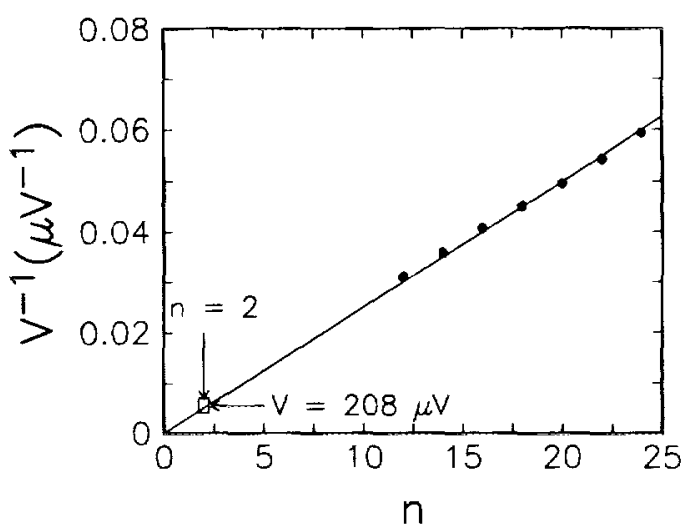

FIG. 3. Plot of Eq. (2) using data shown in Fig. 1(a). The plasma voltage $V_{p}$ is estimated to be $208 \mu \mathrm{V}$ corresponding to a plasma frequency of 104 $\mathrm{GHz}$. 
between the plasma oscillations and the traveling fluxon. The voltages of the FS's are shown to be given by Eq. (1) with $n$ being even integers. Plasma oscillations are also believed to be responsible for of the FS's observed on the higher order steps. Due to complications such as fluxon collisions and fluxon bunching, detailed quantitative calculations need to be done to clarify the situation for higher order steps.

We would like to thank Art Davidson for discussions regarding the role of plasma oscillation in fluxon dynamics.

'T. A. Fulton and R. C. Dynes, Solid State Commun. 12, 57 (1973).

${ }^{2}$ J. T. Chen and D. N. Langenberg, in Low Temperature Physics-LTI3, edited by K. D. Timmerhaus, W. J. O'Sullivan, and E. F. Hammel (Plenum, New York, 1974), Vol. 3, pp. 289-292.

${ }^{3}$ D. W. McLaughlin and A. C. Scott, Phys. Rev. A 18, 1652 (1978).

${ }^{4}$ M. R. Scheuermann, T. V. Rajeevakumar, Jhy-Jiun Chang, and J. T. Chen, Physica B 107, 543 (1981).

5N. F. Pedersen and D. Welner, Phys. Rev. B 29, 2551 (1984).

${ }^{6} B$. Kofoed, J. Mygind, N. F. Pederson, M. R. Samuelsen, D. Welner, and C. A. D. Winther, in Proceedings on the 17th International Conference on Low Temperature Physics, LT-I7, edited by U. Eckern, A. Schmid, W. Weber, and H. Wuhl (North-Holland, New York, 1984), pp. 709-710.

${ }^{7}$ B. Dueholm, E. Joergensen, O. A. Levring, R. Monaco, J. Mygind, N. F. Pedersen, and M. R. Samuelsen, IEEE Trans. Mag. MAG-19, 1196 (1983).

${ }^{8}$ O. A. Levring, N. F. Pedersen, and M. R. Samuelsen, J. Appl. Phys. 54, 987 (1983).

${ }^{9} J$. R. Lhota, M. Scheuermann, P. K. Kuo, and J. T. Chen, IEEE Trans. Mag. MAG-19, 1024 (1983). 